\title{
Impacto en la sociedad del desarrollo del proyecto integrado del cultivo de vainilla orgánica en sistemas agroforestales, y su vinculación al sector agroalimentario
}

Impact on the Society of Implementing the Integrated Project for the Organic Vanilla Cultivation in Agroforestry Systems, and Its Linkage with the Agri-Food Sector

\author{
Amelia Paniagua-Vásquez \\ Universidad Nacional \\ Heredia, Costa Rica \\ amelia.paniagua.vasquez@una.cr \\ José Bernal Azofeifa \\ Universidad Nacional \\ Heredia, Costa Rica \\ jb12azo@gmail.com
}

Recibido: 10/08/2014 Aceptado: 06/27/2016

Resumen: La vainilla es la orquídea económicamente más importante del mundo. Por este motivo, a partir del 2006, el objetivo de la Universidad Nacional, a través de este proyecto fue la implementación de modelos de desarrollo agrarios en sistemas agroforestales (SAF) en regiones socioeconómicamente vulnerables de Costa Rica. Dentro de los principales resultados de esta iniciativa destacan: formación y legalización de la Asociación de Vainilleros Unidos de Costa Rica (APROVAINILLA); curso anual sobre aspectos generales del cultivo de vainilla en SAF con participantes nacionales e internacionales; realización del Primer Seminario Internacional de Vainilla; acceso a fondos del Consejo Nacional para Investigaciones Científicas y Tecnológicas (CONICIT); Fundación para el Fomento y Promoción de la Investigación y Transferencia de Tecnología Agropecuaria en Costa Rica (FITTACORI); Fondo Especial de Educación Superior (FEES); Fondo Institucional de Desarrollo Económico (FIDA) y Fondo Universitario para el Desarrollo Regional (FUNDER); creación del I Banco de Germoplasma del género Vanilla en Costa Rica; alianzas científicas con el Centro de Cooperación Internacional en Investigación Agronó- 
Revista Universidad en DiÁlogo • Vol. 6, N. ², Julio-diciembre 2016, pp. 23-40

ISSN 2215-2849 • EISSN: 2215-4752

DOI: http://dx.doi.org/10.15359/udre.6-2.2

mica para el Desarrollo (CIRAD-Francia); participación estudiantil de diversas carreras del TEC, UNA, UCR, UNED para la realización de tesis de grado y posgrado; producción del documental "Vainilla en Costa Rica"; capacitación de productores en manejo y selección de semilla de vainilla; día de campo por año con productores, estudiantes y miembros de las comunidades; charlas sobre productos no maderables del bosque; participación en seminarios y congresos nacionales e internacionales; exportación de plantas in vitro; publicaciones científicas; obtención de pasaportes científicos. Las experiencias acumuladas en las áreas de extensión, investigación y docencia, las condiciones agroecológicas de Costa Rica, así como el diverso material genético existente abren la posibilidad de desarrollar modelos productivos basados en la agricultura orgánica y, quizá, en el mediano y largo plazo el país podría convertirse en un exportador comercial de vainilla gourmet a nivel mundial.

Palabras clave: vainilla, comunidad, estudiantes, conservación, agricultura orgánica.

Abstract: Vanilla is the most economically important orchid in the world. For this reason, since 2006, the aim of the National University through this project was the implementation of agricultural development models in Agroforestry Systems (AFS) in socioeconomically vulnerable regions of Costa Rica. Among the main results of this project include: formation and legalization of the National Association of Vanilla Producers of Costa Rica (APROVAINILLA), annual course on general aspects of the vanilla cultivation in AFS with national and international participants, realization of the First Vanilla International Seminar, funding of the National Council for Scientific and Technological Research (CONICIT), Foundation for the Development and Promotion of Agricultural Research and Technology Transfer in Costa Rica (FITTACORI), Special Fund for Higher Education (FEES), Institutional Fund of Economic Development (FIDA) and University Fund for Regional Development (FUNDER), establishment of the first Germplasm Bank of Vanilla in Costa Rica, scientific alliances with the Center for International Cooperation in Agronomic Research for Development (CIRAD-France), student involvement of various careers of TEC, UNA, UCR, UNED for conducting graduate and postgraduate thesis, documentary "Vanilla in Costa Rica", training of farmers in managing and selecting vanilla bean, a field day per year with producers, students and community members, speeches on non-timber forest products, participation in national and international seminars and conferences, exporting of in vitro plants, scientific publications, scientific collecting passports. Accumulated experiences in the areas of extension, research and teaching, agroecological conditions in Costa Rica as well as the existing genetic resources, open the possibility to develop productive models based on organic agriculture, and perhaps in the medium - to long-term the country could become a commercial exporter of gourmet vanilla worldwide.

Keywords: vanilla, community, students, conservation, organic agriculture. 
La vainilla (Vanilla planifolia) es la orquídea económicamente más importante del mundo, debido a la producción del compuesto químico vainillina, el cual es la sustancia aromatizante y saborizante de mayor impacto a nivel mundial (Bory, Grisoni, Duval, \& Besse, 2008; Divakaran, Nirmal Babu, \& Peter, 2006; Havkin-Frenkel \& Belanger, 2007; Lubinsky, Bory, Hernández-Hernández, Seung-Chul, \& Gómez-Pompa, 2008; Pak, Gropper, Dai, Havkin-Frenkel, \& Belanger, 2004; Soto, 1999; Sujatha \& Bhat, 2010).

La distribución natural de esta especie comprende los países de México, Guatemala, Belice, Honduras, Costa Rica y Panamá (Schluter, Soto y Harris, 2007; Soto y Cribb, 2010; Soto y Dressler 2010), motivos suficientes para considerar la región como fuente natural de variación genética. A pesar de ello, sus poblaciones naturales se encuentran en peligro de extinción (Soto, 2006). Las principales causas de tal problema son la deforestación, colectas excesivas de material para el establecimiento de nuevas plantaciones y a inadecuados manejos del cultivo (Bory et al., 2008).

De manera que, conociéndose la importancia de la vainilla a nivel mundial y su distribución natural mesoamericana, la Universidad Nacional de Costa Rica, a partir del 2006, inició el proyecto innovador del cultivo de vainilla en sistemas agroforestales $(\mathrm{SAF})$ en regiones económicamente desatendidas de Costa Rica. Los SAF permiten integrar el uso de la tierra, asociándola con cultivos poco tradicionales, de fácil manejo y que generan ingresos económicos adicionales.

En un principio la propuesta se desarrolló con la finalidad de solventar problemas de producción (vainilla y madera) y coadyuvar económicamente a las comunidades participantes; sin embargo, la escasa experiencia sobre el manejo del cultivo y el poco conocimiento de la calidad del material vegetal obligó a replantear la dinámica del proyecto.

Aún así, es necesario ofrecer alternativas para hacer que la actividad forestal sea atractiva y fácil de adoptar por los agricultores/propietarios, quienes tienen poco interés en reforestar. Inclusive Barrantes y Ugalde (2013) mencionaron que, a pesar de que las plantaciones forestales continúan siendo la principal fuente de madera en Costa Rica, en el 2012 se procesó un 5\% menos que en el 2011 y un 34\% menos que en el 2007. Esta tendencia se debe a la disminución de estímulos económicos por parte del Estado, a un alto incremento en el valor de la tierra y, principalmente, a la falta de competitividad que han tenido las plantaciones forestales puras frente a otras actividades productivas, tales como el cultivo de la piña o del banano. 
Revista Universidad en Diálogo • Vol. 6, N. ${ }^{\circ} 2$, Julio-diciembre 2016, pp. 23-40

ISSN 2215-2849 • EISSN: 2215-4752

DOI: http://dx.doi.org/10.15359/udre.6-2.2

Por tanto, es urgente generar alternativas rentables que reduzcan la fuerte presión por los recursos del bosque. A este aspecto, los SAF se convierten en alternativas eficientes para diversificar y darle valor agregado a las fincas.

Un análisis integral de la génesis del proyecto replanteó, durante la ejecución, las acciones prioritarias para conciliar la producción con la conservación de los recursos naturales. En consecuencia, Azofeifa y Paniagua (2014) identificaron aspectos fundamentales para lograr la sustentabilidad y éxito del proyecto. Por una parte, se requiere un manejo eficiente del cultivo por agricultores, productores y campesinos, lo cual demanda eficientes estrategias de extensión para mejorar el compromiso de estos grupos con el proyecto, así como la identificación de personas con “perfil vainillero". Por otra parte, es imperante realizar un diagnóstico de los recursos biológicos de vainilla disponibles en áreas silvestres protegidas y no protegidas del país, pues se halló germoplasma silvestre de vainilla con porcentajes de vainillina más altos de los encontrados en la vainilla gourmet internacional (Azofeifa-Bolaños, Paniagua-Vásquez, \& García-García, 2014).

Hasta la fecha, los avances de los proyectos de vainilla requirieron procesos cíclicos de investigación-extensión para lograr los resultados obtenidos. Por lo tanto, el objetivo de este documento fue describir la importancia de la evolución del proyecto de vainilla orgánica en SAF como mecanismo de cambio en la sociedad y el sector agroalimentario.

\section{Metodología}

La búsqueda de información se realizó de junio a setiembre del 2014. Para ello, este trabajo se fundamentó en la revisión de artículos científicos, libros, capítulos de libros, memorias de seminarios y congresos, tesis, páginas de internet y las experiencias de la Universidad Nacional en la coordinación, planeamiento y ejecución de los siguientes proyectos: “Cultivo y Producción De Vainilla Orgánica en Sistemas Agroforestales en el Asentamiento Campesino Argendora, Santa Cecilia de La Cruz, Guanacaste" (2006-2009), "Desarrollo de Sistemas Agroforestales como alternativa de producción sostenible para pequeños y medianos productores de la zona Atlántica de Costa Rica" (2009-2011), "Mejoramiento del cultivo de vainilla (Vanilla spp.) por medios tradicionales y no tradicionales y su implementación en sistemas agroforestales" (2009-2011) y "Ubicación, caracterización y conservación del germoplasma de vainilla presente en Costa Rica" (20122014). La redacción del documento se realizó de enero a mayo del 2013. 


\section{Resultados y discusión}

Los resultados más sobresalientes de los proyectos de vainilla en los cuales la Universidad Nacional participó, ya fuese como coordinadora o coadyuvante de otras instituciones, se detallan en los subsiguientes párrafos.

\section{Asociación NacionaldeVainillerosUnidosdeCostaRica(APROVAINILLA)}

APROVAINILLA fue creada en el año 2007 como iniciativa de varias personas entusiastas interesadas en la producción e industrialización de un producto innovador para el ámbito local. Este entusiasmo surgió gracias a la información recibida en los cursos impartidos por el Instituto de Investigación y Servicios Forestales (INISEFOR) en conjunto con la Escuela de Ciencias Agrarias de la Universidad Nacional de Costa Rica (UNA). Sin embargo, no fue sino hasta el 2009 cuando la asociación se constituyó legalmente, con una visión, misión y objetivos estratégicos definidos (http://www.aprovainilla.org/).

Actualmente, está integrada por más de 40 asociados. A través de este ente se realizan capacitaciones constantes, cursos, talleres, visitas a campo y, recientemente, en la página electrónica se encuentran boletines técnicos de acceso libre para complementar la ayuda a los productores, satisfacer a los consumidores y consolidar en un futuro cercano un producto de calidad (http://www.aprovainilla.org/). Aunado a ello, se ofrece la oportunidad de acompañar a los agricultores/productores a ingresar a un mercado en crecimiento sin comprometer el equilibrio natural del medio ambiente.

\section{Curso anual sobre el cultivo de vainilla orgánica en SAF}

Desde el 2006, esta iniciativa se desarrolló con la finalidad de acercar la academia con el sector productivo. Actualmente, para formar parte de APROVAINILLA y recibir asesoría técnica por parte de expertos de la Universidad Nacional, es necesario concluir con éxito el programa del curso.

La capacitación -inicialmente formulada para participantes costarricenses- se proyectó a nivel internacional y, a partir del 2014, recibió participantes de Perú, Colombia y Estados Unidos. Esta brinda a los participantes los conceptos básicos del funcionamiento de las plantas de vainilla, la respuesta y necesidades a las condiciones ambientales y nutricionales, su relación en el sistema agroforestal (SAF) y la optimización de estos recursos en la productividad. También se incluye el crecimiento y el desarrollo de las plantas dentro de un SAF, con el 
Revista Universidad en Diálogo • Vol. 6, N. ${ }^{\circ} 2$, Julio-diciembre 2016, pp. 23-40

ISSN 2215-2849 • EISSN: 2215-4752

DOI: http://dx.doi.org/10.15359/udre.6-2.2

fin de poder realizar un manejo eficiente del sistema. Posteriormente, el curso se complementará en aspectos de manejo post cosecha de la vainilla.

El objetivo general del curso es profundizar en el conocimiento de aspectos morfológicos, fisiológicos y ambientales del cultivo de la vainilla en SAF. Por su parte, los objetivos específicos son: describir los SAF asociados al cultivo de la vainilla, describir los principios taxonómicos y biología reproductiva del cultivo de la vainilla (condiciones agroecológicas ideales), describir la morfología de la planta de vainilla (raíz, tallo, hoja, flor y fruto), describir los microorganismos endófitos y entomopatógenos de la vainilla, describir las necesidades nutricionales del cultivo de la vainilla, describir las condiciones de comercialización de la agricultura orgánica y conocer los potenciales mercados nacionales e internacionales para su comercialización.

\section{Seminario Internacional de Vainilla en Costa Rica}

Durante los días del 4 al 8 de noviembre del 2013 se celebró el Primer Seminario Internacional de Vainilla en Heredia, Costa Rica. El evento reunió a investigadores, académicos, empresarios, productores, estudiantes, y representantes gubernamentales de Costa Rica, Panamá, Perú, México, Honduras, Estados Unidos, Alemania y Francia.

Los objetivos del seminario fueron dar a conocer las experiencias de trabajo a nivel nacional e internacional en investigación, extensión y producción de Vanilla spp., y crear alianzas y sinergias de trabajo intra e interinstitucional a nivel nacional e internacional. Para ello, se utilizó una estrategia de comunicación, la cual consistió en el envío directo de invitaciones a través de correos electrónicos, llamadas telefónicas y la creación de un portal electrónico con información general del seminario, el lugar del evento, los objetivos, las temáticas, el cronograma, los expositores, el comité evaluador, el comité científico, los procedimientos de inscripción, el instructivo para los autores sobre el envío de resúmenes, las fechas importantes, los hoteles para hospedaje y la persona contacto (http://seminariovainillacrc.wix.com/semvainillacrc2013).

Dentro de las temáticas abordadas en el evento se destacaron las siguientes: conservación de Vanilla spp., mejoramiento genético, taxonomía, plagas y enfermedades; bioprospección de parientes silvestres y cultivados; micro y macropropagación; importancia económica, ecológica y social; fermentación y curado; cultivo de vainilla en SAF; invernaderos u otro sistema de cultivo; costos de producción, análisis económicos y financieros; mercado de 
la vainilla; densidad de siembra óptima; demanda hídrica y control biológico

Para esta primera reunión se contó con la participación de cinco conferencistas magistrales, todos con amplia experiencia en el trabajo con vainilla. Estos fueron: Michel Grisoni (Francia), Braulio Edgar Herrera Cabrera (México), Juan Hernández Hernández (México), Delfino Reyes López (México) y Henry Arturo Kelso Bucio (Panamá). Además, se contó con un comité evaluador de los resúmenes y documentos a texto completo para la memoria final. Se recibieron un total de 32 resúmenes, de los cuales se aceptaron 16 y 12 se enviaron a texto completo. La publicación del libro de memorias se realizará a finales del 2014.

\section{Proyectos financiados}

Desde su visualización en el 2006, la vainilla generó varios proyectos en los cuales se destacó el planeamiento, la consecución de fondos de diferentes entidades financiadoras, la coordinación y ejecución de proyectos integrados interinstitucionales y multidisciplinarios. Estos lograron el conocimiento y las habilidades en investigación y extensión necesarias para ahondar en procesos más específicos y necesarios relacionados con la conciliación de la producción (mejoramiento de la calidad de vida) con la conservación del patrimonio vainilla. A continuación, los proyectos:

\section{Cultivo y producción de vainilla (Vanilla fragans) orgánica, asociada a dos especies forestales en la provincia de Guanacaste (2006-2008)}

La primera iniciativa relacionada con vainilla fue financiada por la Fundación para el Fomento y Promoción de la Investigación y Transferencia de Tecnología Agropecuaria en Costa Rica (FITTACORI). Dentro de los resultados más destacados de este proyecto se indican la selección de 12 fincas donde se realizaron ensayos del cultivo de vainilla en asocio con árboles de caoba (Swietenia macrophylla King), laurel (Cordia alliodora (Ruiz \& Pav.) Cham) y Neem (Azadirachta indica A. Juss). En cada parcela seleccionada se cultivaron 250 plantas procedentes del cultivo in vitro. También, se identificó el impacto socioeconómico y ambiental de las condiciones de vida de los productores y sus familias con la producción de vainilla orgánica, por medio de metodologías participativas y análisis financiero de la producción de vainilla orgánica. Finalmente, se capacitó a 60 personas sobre el cultivo en SAF mediante la realización de un taller de impacto ambiental y prácticas conservacionistas. 
Revista Universidad en Diálogo • Vol. 6, N. ํㄹ 2, Julio-diciembre 2016, pp. 23-40

ISSN 2215-2849 • EISSN: 2215-4752

DOI: http://dx.doi.org/10.15359/udre.6-2.2

Desarrollo de capacidades en la comunidad de Argendora mediante el cultivo de la vainilla (Vanilla sp.) en sistemas agroforestales (Enero a diciembre, 2009)

Este proyecto fue financiado por el Fondo Universitario para el Desarro1lo Regional de la Universidad Nacional (FUNDER) y ejecutado por el INISEFOR y el Centro Mesoamericano de Desarrollo del Trópico Seco (CEMEDE) de la Sede Regional Chorotega.

Este implementó dos fincas demostrativas para 21 participantes, los cuales aprendieron sobre la funcionalidad de los SAF y el manejo agronómico de la vainilla en dichos sistemas de cultivo. Además, se analizaron las perspectivas agroforestales y turísticas de la comunidad de Argendora en La Cruz de Guanacaste (Sánchez \& Jiménez, 2012).

Fortalecimiento de la organización comunal y desarrollo de capacidades empresariales en los pequeños productores de las comunidades fronterizas del cantón de La Cruz. Guanacaste

Con el Fondo Investigación y Desarrollo Académico (FIDA) y en colaboración con la sede Chorotega y el CEMEDE, se trabajó en esta iniciativa, en el año 2010. Entre sus principales logros estuvo la participación e involucramiento de más de 150 personas en el cantón implementando actividades productivas asociadas a la principal, entre esas personas participaron los productores de vainilla. Hoy día en la comunidad de Argendora, junto a otras instituciones, han elaborado e implementado una ruta que han llamado turismo ecológico.

Proyectos de vainilla financiados por el Fondo Especial de Educación Superior (FEES) del Consejo Nacional de Rectores (CONARE), en conjunto con otras universidades

Desarrollo de capacidades en los pequeños y medianos productores en sistemas agroforestales (SAF) con el cultivo y producción de vainiIla (Vanilla sp.) de la zona Atlántica. (Enero a diciembre 2009). (CRI)

Entre los principales logros de esta iniciativa destacan la identificación y ubicación de seis fincas con perfil para el trabajo con vainilla en SAF. En ellas se aplicaron muestreos físicos, químicos y microbiológicos a 10 lotes. Del análisis se concluyó que los suelos de todas las fincas son de origen 
aluvial (Inceptisoles) y aptos para la actividad agropecuaria, bien drenados aunque susceptibles a la compactación.

Además, se capacitó a 22 personas en SAF mediante la realización de un taller y se establecieron tres fincas en las cuales se desarrolló un modelo de SAF con poró (Erythrina sp.) y madero negro (Gliricidia sepium (Jacq.) Kunth ex Walp) como tutores y asociados con árboles de cacao (Theobroma cacao L.) y vainilla. Esta iniciativa originó otro proyecto de SAF en el Atlántico.

Desarrollo de sistemas agroforestales como alternativa de producción sostenible para pequeños y medianos productores de la zona Atlántica de Costa Rica (2009-2011)

Este proyecto logró establecer un modelo de sistema productivo al integrar componentes forestales y agrícolas. El diseño de SAF consistió en el cultivo de tutores de poró y madero negro con árboles de roble coral (Terminalia amazonia (J.F. Gmel.) Exell), chancho (Vochysia guatemalensis Donn. Sm.) y cacao. Se realizaron capacitaciones sobre el cultivo de dichas especies con vainilla en SAF. Las plantas de vainilla para dicho sistema procedieron de plantas in vitro y esquejes.

También, se evaluó la incidencia de la instalación del sistema productivo en los aspectos financiero y productivo. Finalmente, se identificaron y monitorearon las principales plagas y enfermedades presentes en los SAF en asocio con el cultivo de vainilla.

Cultivo y producción de vainilla orgánica en sistemas agroforestales en el asentamiento campesino Argendora, Santa Cecilia de La Cruz, Guanacaste (2006-2009)

Esta propuesta integrada (investigación, extensión y docencia) se diseño para incrementar y mejorar las condiciones de vida de los productores y las familias de Argendora en La Cruz, Guanacaste, por medio de la implementación de SAF, utilizando para ello, la vainilla como cultivo promisorio. El sistema instalado se diseñó con una visión de producción orgánica debido a los altos precios y al excelente mercado a nivel nacional e internacional de sus productos (especialización de mercados); además, se incorporó el conocimiento y la implementación de tecnologías de mínimo impacto, amigables con el ambiente y en asocio con especies forestales de alto valor. Finalmente, se ofreció una alternativa estable de trabajo para personas comprometidas con el cultivo, la sociedad y su comunidad. 
Revista Universidad en Diálogo • Vol. 6, N. ํㄹ 2, Julio-diciembre 2016, pp. 23-40

ISSN 2215-2849 • EISSN: 2215-4752

DOI: http://dx.doi.org/10.15359/udre.6-2.2

Si bien es cierto, en Argendora no se alcanzó el 100\% de las producciones esperadas, se logró que los pobladores adoptarán, por vez primera, el SAF con vainilla. Aunque en los inicios muchos pequeños productores no creyeron en el proyecto y no siguieron con rigurosidad las buenas prácticas agrarias que el cultivo requería, al pasar de los años observaron fructificaciones de forma natural y comentaron la alegría de observar por vez primera ese proceso y, a la vez, reconocieron que en esa primera floración no obtuvieron los rendimientos esperados debido a la falta de acato de los lineamientos previamente indicados en los talleres impartidos por los investigadores de la UNA (Azofeifa \& Paniagua, 2014). En consecuencia, en la zona existen vainilleros aptos para multiplicar la experiencia vivida y acompañar a futuros agricultores/productores en el proceso.

La experiencia que se generó durante los primeros cuatro años del proyecto originó el manual titulado "Vainilla orgánica en SAF", el cual brinda amplios conceptos sobre el manejo de la vainilla en SAF, entre otras temáticas vinculadas al cultivo (Paniagua \& García, 2009).

\section{Mejoramiento del cultivo de vainilla (Vanilla spp.) por medios tradicionales y no tradicionales y su implementación en sistemas agroforestales" (2009-2011). (FEES)}

Esta iniciativa en coordinación con el TEC en Santa Clara, era la implementación en un sistema agroforestal de plantas in vitro creadas por medio de la fusión de protoplastos, estas nuevas plantas no se lograron obtener en el tiempo estipulado en el proyecto. Sin embargo, se trabajó y llevaron al campo plantas producidas in vitro años atrás y actualmente están creciendo en un ambiente completamente natural. La idea es evaluar la producción e identificar los genes para describir esta planta que se ha difundido a nivel nacional. Para ello se debe esperar la producción, proceso que aun no inicia, ya que la vainilla es una planta que requiere de al menos 4 años para alcanzar su madurez fisiológica

\section{Ubicación, caracterización y conservación del germoplasma de vainilla presente en Costa Rica (setiembre 2012-setiembre 2014)}

Los proyectos integrados mencionados anteriormente originaron esta investigación. Esta fue financiada por el Consejo Nacional para Investigaciones Científicas y Tecnológicas (CONICIT). Dentro de sus principales logros se destacó la revisión bibliográfica "Importancia y desafíos de la conser- 
vación de Vanilla spp. (Orquidaceae) en Costa Rica" (Azofeifa-Bolaños et al., 2014) y el artículo científico "Caracterización morfométrica de tallos y hojas de Vanilla spp. (Orchidaceae) de Costa Rica mediante análisis multivariado" el cual se encuentra en edición en una revista especializada.

Se estableció un protocolo de colecta para vainilla. Este consistió, en primer lugar, en la ubicación a través del uso de sistemas de información geográfica de las plantas en su estado natural y, en segundo lugar, el traslado de las muestras en buenas condiciones desde los sitios de colecta hasta su establecimiento en invernadero, donde se trabajó la capacidad y adaptabilidad fisiológica.

Se recolectaron muestras de herbario de los tutores naturales y se creó la primera lista de especies donde la vainilla crece naturalmente (árboles, arbustos, hierbas, helechos y palmas).

Se encontraron poblaciones silvestres nunca antes registradas dentro y fuera de áreas silvestres protegidas y producto del mapeo de las zonas donde se encontraron especímenes, se generó una base de datos actualizada, con sus respetivos mapas de distribución.

Este proyecto generó la colección de más de 200 accesiones, de las cuales 125 se caracterizaron morfométricamente en el primer banco de germoplasma del género en Costa Rica. El análisis multivariado, así como el conocimiento biológico del género discriminó 20 grupos con coincidencias en sus características morfométricas, lo cual evidencia, por una parte, la diversidad morfométrica de la hoja de vainilla para discriminar germoplasma y, por otra parte, la enorme diversidad de especies de vainilla en los bosques costarricenses.

\section{Banco de germoplasma del género vanilla en Costa Rica}

La vainilla está sujeta a fuertes presiones de selección al ser considera la orquídea económicamente más importante del mundo. Sus poblaciones naturales se reducen debido a la deforestación, la excesiva recolección para el establecimiento de nuevas plantaciones y a un pobre manejo del cultivo (Bory et al., 2008). Aunado a ello, durante la ejecución de los proyectos de producción de vainilla en SAF, surgieron muchas interrogantes técnicas, las cuales debieron abordarse con enfoques investigativos. Entre estas: cuál es el material genético con que cuenta el país, cuál es la calidad del material cultivado por los productores y lo fundamental, qué pasará con estos en el futuro próximo. 
Revista Universidad en Diálogo • Vol. 6, N. ํㄹ 2, Julio-diciembre 2016, pp. 23-40

ISSN 2215-2849 • EISSN: 2215-4752

DOI: http://dx.doi.org/10.15359/udre.6-2.2

Por todo lo comentado, para solucionar los problemas de erosión genética del género y conocer el material genético costarricense fue necesario priorizar una estrategia nacional de conservación. De esta forma, la Universidad Nacional de Costa Rica obtuvo financiamiento por parte del Consejo Nacional para Investigaciones Científicas y Tecnológicas (CONICIT) y, a partir del 2012, estableció el primer banco de germoplasma en invernadero, el cual es el lugar más adecuado para un género cuya forma predominante de reproducción es asexual (crecimiento vegetativo) (Azofeifa-Bolaños et al., 2014)

El establecimiento de bancos de germoplasma a partir de semillas en vainilla no se recomienda, debido a la germinación errática. Las semillas son muy pequeñas, presentan un embrión indiferenciado, tegumentos muy duros y cerosos que contienen inhibidores de germinación (Bory et al., 2008).

Por tanto, esta iniciativa espera ampliar la base genética del género y reducir significativamente la vulnerabilidad de extinción de algunas de sus especies.

Se han encontrado poblaciones silvestres nunca antes registradas dentro y fuera de áreas silvestres protegidas. Hasta la fecha, la colección cuenta con más de 200 accesos de vainilla silvestre y cultivada, la cual se encuentra parcialmente caracterizada a nivel morfológico y molecular. Se estima que existen parientes silvestres no descubiertos, así como nuevas especies no registradas.

\section{Alianzas científicas con el Centro de Cooperación Internacional en Investigación Agronómica para el Desarrollo (CIRAD), Francia}

La realización del I Seminario Internacional de Vainilla en Costa Rica hizo eco en la comunidad científica nacional e internacional. De esa forma, se empezó a gestionar un acuerdo de transferencia de material (MTA) con la sede del CIRAD en la isla de La Reunión, Francia, con la finalidad de realizar, en una primera etapa, trabajos científicos integrales y sistemáticos sobre la diversidad genética de las vainillas costarricenses, así como la biología reproductiva de poblaciones naturales. Nuestro grupo de investigación identificó poblaciones silvestres de Vanilla pompona en Costa Rica. Estos hallazgos permitieron el trabajo en conjunto de la UNA con el CIRAD y, como resultado de dicha alianza, se está preparando un artículo científico sobre la diversidad biológica del complejo Vanilla pompona, para ser publicado a principios del 2015 en una revista especializada de alto impacto. 


\section{Tesis de grado y postgrado}

La visualización de los proyectos de vainilla por la comunidad estudiantil generó y continúa con la generación de investigaciones y propuestas de trabajos de graduación para optar por títulos universitarios de grado y postgrado. Entre las experiencias generadas se destacan las siguientes:

\section{Trabajos de graduación concluidos}

Corrales, Y., Eduarte, E. (2010). Propuesta para el mejoramiento e incorporación de los sistemas agroforestales como una alternativa de producción a pequeños productores en el asentamiento campesino Argendora en La Cruz de Guanacaste (Tesis de licenciatura). Universidad Nacional de Costa Rica, Heredia, Costa Rica.

Montoya, R. (2010). Comparación genética de diferentes genotipos de vainilla en Costa Rica. (Tesis de bachillerato). Universidad Nacional de Costa Rica, Heredia, Costa Rica.

Rodríguez-Hernández, M. G. (2013). Crecimiento y multiplicación de vanilla planifolia bajo condiciones de un sistema de inmersión temporal (Tesis de bachillerato). Universidad Nacional de Costa Rica, Heredia, Costa Rica.

Valderrama-Ordoñez, M. G. (2014). Caracterización morfológica y propagación asexual in vitro de Vanilla sp., procedente de Parismina (Tesis de bachillerato). Universidad Nacional de Costa Rica, Heredia, Costa Rica.

\section{Trabajos de graduación en ejecución}

Azofeifa, J. B. Respuestas morfogenéticas de plántulas in vitro y esquejes de Vanilla planifolia Andrews cultivadas en invernadero y en Sistemas Agroforestales, Guápiles, Costa Rica (Tesis de maestría). Universidad Estatal a Distancia. San José, Costa Rica.

Azofeifa, M. A. Evaluación agroecológica de un modelo de sistema agroforestal que incluye teca, melina y chicasquil asociado a poró como tutor en el crecimiento inicial de Vanilla planifolia, San Carlos, Costa Rica (Tesis de licenciatura). Universidad Nacional de Costa Rica, Heredia, Costa Rica.

Jiménez, J. J. Producción en verde de vainilla orgánica en un sistema agroforestal (Tesis de licenciatura). Universidad Estatal a Distancia. San José, Costa Rica. 
Revista Universidad en Diálogo • Vol. 6, N. ํㄹ 2, Julio-diciembre 2016, pp. 23-40

ISSN 2215-2849 • EISSN: 2215-4752

DOI: http://dx.doi.org/10.15359/udre.6-2.2

Sánchez, M. M. Caracterización morfológica y propagación asexual de Vanilla spp mediante el uso de reguladores de crecimiento (Tesis de licenciatura). Universidad Nacional de Costa Rica, Heredia, Costa Rica.

\section{Documental de vainilla}

En mayo del 2013 se transmitió en la Universidad Nacional el documental Vainilla en Costa Rica. Este video se grabó en un sistema de alta definición y tiene una duración de 39 min. Resume de forma gráfica el alcance de los diferentes proyectos de la vainilla en las localidades de Argendora, La Cruz; La Colonia, Guápiles, y Sagrada Familia, Cariari. De esa manera, se narraron las historias de las personas involucradas con el cultivo de la vainilla, las cuales, con sus propias palabras, relataron las actividades sobre el manejo agrobiológico de sus plantaciones. Además, se hizo un repaso sobre la evolución del proyecto y se finalizó con una reseña de la importancia que tiene el proyecto en términos ecosistémicos y la visión conciliadora entre el uso apropiado de un recurso natural para generar calidad de vida y el respecto con la conservación de su medio ambiente.

\section{Otros resultados}

- La búsqueda sistemática de regiones agroclimáticas adecuadas para el cultivo de vainilla en SAF y con bajos índices de desarrollo determinó a las comunidades de Argendora, La Cruz; La Colonia, Guápiles; Bocana, Puriscal; San Carlos; Pérez Zeledón y Quepos, como lugares adecuados para la implementación de modelos agroforestales con vainilla.

- Otro aspecto importante de puntualizar fue la capacitación constante de los productores a través de talleres aplicando metodologías horizontales inclusivas (aprendiendo haciendo), donde se explicó el manejo y selección de la semilla de vainilla por finca. Además, se realizaron días de campo, uno por año, para conocer la experiencia de cada productor en el proceso. Esta actividad contó con la participación de algunos miembros de la comunidad y los estudiantes de la escuela y colegio cercanos.

- En relación con la proyección del proyecto, se participó en seminarios y congresos nacionales e internacionales sobre la experiencia generada (Cuba, Uruguay y Costa Rica). El aprendizaje que se generó se conceptualizó en la redacción de nuevas propuestas de proyectos, generación de contactos y conceptualización del alcance al cual es posible llegar con esta innovación de cultivo en SAF con vainilla. Así mismo, se escribieron 
los siguientes artículos Investigación y extensión, ¿son complementarias? Caso vainilla (Azofeifa y Paniagua, 2014) y La experiencia del trabajo de vainilla en SAF (Paniagua y Azofeifa, 2014). Ambos se publicarán en una revista de extensión de la Universidad Nacional de Costa Rica.

- Como parte de las estrategias para la conservación ex situ del germoplasma de vainilla costarricense se establecieron esquejes de $V$. planifolia colectados de diferentes partes del país a través de la técnica de cultivo de tejidos (in vitro). La optimización de protocolos genotipo-específicos es necesario de realizar para las diferentes especies de vainilla y tener un duplicado del germoplasma en laboratorio, pues el manejo en vivero para algunas de ellas se complica debido a la escasez de raíces. Una vez establecidos en condiciones heterótrofas, se multiplicaron masivamente, se aclimataron en invernadero y, finalmente, se establecieron en campo. De este material, se exportaron cinco mil plantas, la primera venta de servicios a otro país.

- Para la búsqueda sistemática de germoplasma silvestre de vainilla en Costa Rica, fue necesario ingresar a las áreas silvestres protegidas. Por motivos éticos y científicos, se obtuvieron los pasaportes científicos para realizar colectas de material vegetal, a través del Sistema Nacional de Áreas de Conservación (SINAC) (Resolución Nº 061-2013; Resolución N 071-2014).

- Finalmente, se realizaron charlas sobre productos no maderables del bosque (PNMB) y un curso anual sobre producción y comercialización de vainilla orgánica en SAF. Desde el año 2013, este evento se realiza en conjunto con el Programa de Capacitación del Colegio de Ingenieros Agrónomos de Costa Rica, lo cual significó un avance muy importante en el trabajo logístico y en la vinculación entre instituciones.

\section{Impacto en la sociedad}

Durante la década de 1970, el Ministerio de Agricultura y Ganadería (MAG) inició un programa de vainilla con productores del sur de Quepos. Sin embargo, por circunstancias gubernamentales, los técnicos encargados de dar asistencia fueron reubicados a otras regiones. En el traslado, los programas y proyectos que se ejecutaban quedaron a la deriva, así como los agricultores. La carencia de asistencia técnica ocasionó enfermedades en las plantas y pérdidas de los vainillales. Los agricultores, que apenas iniciaban a conocer este cultivo, quedaron defraudados. Casi 20 años después, la UNA inició un proyecto con la recomendación de uno de los técnicos del MAG, persona consciente de las bondades económicas, sociales y ambientales de la vainilla. 
Paralelo al proyecto, varias personas solicitaron información sobre el cultivo de vainilla, porque desde el inicio se le dio una gran difusión. Debido a esas solicitudes de información, se ofreció un curso básico sobre el cultivo, el cual se repite anualmente. Estos cursos se iniciaron en las instalaciones del INISEFOR en Heredia, luego se proyectaron a diferentes comunidades y actualmente se imparten en las comunidades y, una vez al año, en las instalaciones del Colegio de Ingenieros Agrónomos de Costa Rica en Moravia. Producto de los asistentes al curso, se formó un grupo de productores, quienes decidieron agruparse en una asociación de acuerdo con la ley 218 con el nombre de: Asociación Nacional de Vainilleros Unidos (APROVAINILLA 3-002-582-758). Se buscó información sobre el cultivo de vainilla y se encontró poca y poco consistente; mucho de lo que se sabe es transmitido en forma oral entre las personas. Pero lo escrito, con evidencia y fundamento es muy escaso, por lo que este es el primer reto: investigar y publicar.

\section{Pasantía de estudiantes}

Durante la ejecución en este proyecto se ha contado con la participación de estudiantes de colegios técnicos profesionales que hacen su práctica supervisada, así como estudiantes de universidades de Cuba, México, Honduras. La participación estudiantil ha sido un aporte muy importante para el desarrollo de estos proyectos.

\section{Conclusiones}

Los resultados generados en los diferentes proyectos de vainilla a través de los primeros 9 años de ejecución justifican el rescate y conservación del germoplasma de Vanilla spp., de Costa Rica, con la finalidad de incorporar estos genes de interés en material genético comercial y para los futuros programas de fitomejoramiento. El material resultante se entregará a las comunidades agrarias con bajos índices socioeconómicos.

Las condiciones agroecológicas de Costa Rica, así como el recurso genético vainilla existente, generarán modelos productivos y especializados basados en la agricultura orgánica. De esa forma, se espera posicionar en el mediano y largo plazo a Costa Rica como un exportador de vainilla gourmet a nivel mundial. 


\section{Referencias}

Azofeifa, J. B. y Paniagua, A. (2015). Investigación y extensión, ¿son complementarias? Caso vainilla. Universidad En Diálogo, 5(1), 73-86.

Azofeifa-Bolaños, J. B., Paniagua-Vásquez, A., y García-García, J. A. (2014). Importancia y desafíos de la conservación de Vanilla spp. (Orquidaceae) en Costa Rica. Agronomía Mesoamericana, 25(1), 189-202. Recuperado de http://revistas.ucr.ac.cr/index.php/ agromeso/article/view/14220

Barrantes, A., y Ugalde, S. (2013). Usos y aportes de la madera en Costa Rica: Estadísticas 2012. En A. Barrantes \& S. Ugalde, Eds., (p. 32). San José, Costa Rica: Oficina Nacional Forestal. Recuperado de http://onfcr.org/media/uploads/documents/onf_usosaportes_2.pdf

Bory, S., Grisoni, M., Duval, M.-F., y Besse, P. (2008). Biodiversity and preservation of vanilla: Present state of knowledge . Genetic Resources and Crop Evolution, 55, 551-571. doi:10.1007/s10722-007-9260-3

Divakaran, M., Nirmal Babu, K., y Peter, K. V. (2006). Conservation of Vanilla species, in vitro. Scientia Horticulturae, 110, 175-180. doi:10.1016/j.scienta.2006.07.003

Havkin-Frenkel, D., y Belanger, F. C. (2007). Application of metabolic engineering to vanillin biosynthetic pathways in Vanilla planifolia. In R. Verpoorte, A. W. Alfermann, \& T. S. Johnson (Eds.), Applications of Plant Metabolic Engineering (pp. 175-196). Springer Netherlands.

Lubinsky, P., Bory, S., Hernández-Hernández, J., Seung-Chul, K. y Gómez-Pompa, A. (2008). Origins and Dispersal of Cultivated Vanilla (Vanilla planifolia Jacks. [Orchidaceae]) . Economic Botany, 62(2), 127-138.

Pak, F. E., Gropper, S., Dai, W. D., Havkin-Frenkel, D., y Belanger, F. C. (2004). Characterization of a multifunctional methyltransferase from the orchid Vanilla planifolia . Plant Cell Reports, 22, 959-966. doi:10.1007/s00299-004-0795-x

Paniagua, A., y García, J. (2009). Manual para el cultivo de vainilla en sistemas agroforestales. (A. Paniagua Vásquez \& J. A. García García, Eds.) (p. 76). Heredia, Costa Rica: Universidad Nacional. Instituto de Investigación y Servicios Forestales.

Sánchez, N., y Jiménez, L. (2012). Procesos universitarios para el fortalecimiento del desarrollo regional: El caso del fondo concursable FUNDER. Universidad En Diálogo, 2(1), 1-30. Recuperado de http:/www.revistas.una.ac.cr/index.php/dialogo/article/view/5630

Schluter, P., Soto, M., y Harris, S. (2007). Genetic variation in Vanilla planifolia (Orchidaceae) . Economic Botany, 61(4), 328-336. 
Revista Universidad en Diálogo • Vol. 6, N. ํㄹ 2, Julio-diciembre 2016, pp. 23-40

ISSN 2215-2849 • EISSN: 2215-4752

DOI: http://dx.doi.org/10.15359/udre.6-2.2

Soto, M. (1999). Filogeografía y recursos genéticos de las vainillas de México. México D. F.: Instituto Chinoin AC, Herbario de la Asociación Mexicana de Orquideología.

Soto, M. (2006). La vainilla: Retos y perspectivas de su cultivo. Biodiversitas, 66, 1-9.

Soto, M., y Cribb, P. (2010). A new infrageneric classification and synopsis of the genus Vanilla Plum. ex Mill. (Orchidaceae: Vanillinae) . Lankesteriana, 9(3), 355-398.

Soto, M., y Dressler, R. (2010). A revision of the Mexican and Central American species of Vanilla Plumier ex Miller with a characterization of their its region of the nuclear ribosomal DNA . Lankesteriana, 9(3), 285-354.

Sujatha, S., y Bhat, R. (2010). Response of vanilla (Vanilla planifolia A.) intercropped in arecanut to irrigation and nutrition in humid tropics of India . Agricultural Water Management, 97(7), 988-994. doi:10.1016/j.agwat.2010.01.031 